\title{
A Solid State Nanopore Device for Investigating the Magnetic Properties of Magnetic Nanoparticles
}

\author{
SangYoon Park ${ }^{1, *}$, Jaekwan Lim ${ }^{1}$, Y. Eugene Pak ${ }^{1}$, Seunghyun Moon ${ }^{2}$ and Yoon-Kyu Song ${ }^{1,2, *}$ \\ 1 Advanced Institutes of Convergence Technology, Suwon 443-270, Korea; \\ E-Mail: jakelim09@gmail.com (J.L.); genepak@snu.ac.kr (Y.P.) \\ 2 Department of Transdisciplinary Studies, Seoul National University, Seoul 151-742, Korea; \\ E-Mail: irus7711@snu.ac.kr \\ * Authors to whom correspondence should be addressed; E-Mail: yoonpark77@snu.ac.kr (S.P.); \\ songyk@snu.ac.kr (Y.S.); Tel.: +82-31-888-9133; Fax: +82-31-888-9148.
}

Received: 29 March 2013; in revised form: 14 April 2013 / Accepted: 17 May 2013 /

Published: 24 May 2013

\begin{abstract}
In this study, we explored magnetic nanoparticles translocating through a nanopore in the presence of an inhomogeneous magnetic field. By detecting the ionic current blockade signals with a silicon nitride nanopore, we found that the translocation velocity that is driven by magnetic and hydrodynamic forces on a single magnetic nanoparticle can be accurately determined and is linearly proportional to the magnetization of the magnetic nanoparticle. Thus, we obtained the magneto-susceptibility of an individual nanoparticle and the average susceptibility over one hundred particles within a few minutes.
\end{abstract}

Keywords: nanopore; magnetic nanoparticle; ion current blockade; magneto-susceptibility

\section{Introduction}

The most important issue for magnetic materials is an understanding of the magnetic and physical properties of a nanometer-sized particle because the use of magnetic nanoparticles (MNPs) in many applications depends predominantly on their inherent magnetic properties. In the case of biomedical applications, the forces that influence the translational, rotational, and vibrational motion of a MNP- tagged biomolecule are the magnetic force due to the magnetization of an individual MNP and the external magnetic field under a given condition [1-3]. Popular magnetic biosensing platforms such 
as giant magneto-resistance (GMR) sensors, Hall sensors, and magneto-optical sensors that are used to quantitatively analyze the existence of target molecules are based on the detection of a stray field from a single MNP [4-7]. Furthermore, structural assemblies of MNPs form various recording media and permanent magnets [8].

Recently, there have been many attempts to quantitatively analyze a biomolecule by magnetically manipulating the motion of magnetic beads in a microfluidic system because magnetic interactions are generally not affected by changes in the surface charges, $\mathrm{pH}$, or ionic concentration of the surrounding medium, contrary to electrical-driven manipulation [9].

To precisely manipulate the motion, we should know the characteristics of the MNP, e.g., the size, charge state, and magnetism. There are well-established techniques for investigating the physical and electrical properties of a nanoparticle. For instance, the size and surface charge state of a nanoparticle can be routinely characterized by dynamic light scattering (DLS) and zeta-potentiometry, respectively. These methods can provide information on the distributions and the average values of the size and charge number of each particle. Magnetic characteristics have been usually determined by a superconducting quantum interference device (SQUID) and by a vibrating sample magnetometer (VSM). Unlike DLS and zeta-potentiometry, these techniques, although they have excellent sensitivities, are limited to the average properties of a large number of MNPs. To the best of our knowledge, the resultant data from these techniques do not provide sufficient information on the magnetism of a MNP, which may be considered as a critical bottleneck in facilitating the use of MNPs in various industrial applications. Therefore, the need to characterize a single MNP has been increasingly recognized.

In this work, we investigate the motion of MNPs, $30 \mathrm{~nm}$ in diameter, that are driven by both magnetic and electric forces in a nanopore membrane. In this investigation, we measured the velocity of the MNPs passing through the nanopore using an ionic current blockade. We found that the magnetic force enables the MNPs to move more rapidly and that the velocity is linearly proportional to the magnetization of a MNP. Thus, we were able to measure the magnetization of a single colloidal MNP and to acquire hundreds of data points on the magnetization within a few minutes.

\section{Methods}

A nanopore chip, which has a single nanometer-sized hole in a free-standing ultrathin membrane between two miniaturized fluidic chambers ("cis" and "trans"), is hydrated by conducting a fluid through it. An electric potential is applied across the chip; an electric current is used because the conduction of ions through the nanopore can be observed. When MNPs with negative charges are immersed in the cis chamber, they are captured and linearly threaded through the nanopore from the cis to trans chamber, as shown in Figure 1, and the current between the two electrodes is momentarily interrupted, which is the so-called "ionic current blockade". The magnitude $\left(\Delta \mathrm{I}_{\mathrm{C}}\right)$ and dwell-time $(\Delta \mathrm{t})$ of the current blockade are governed by the size of the particle under given conditions [10]:

$$
\Delta I_{C}=\left[S\left(d_{C}, d_{S}\right) \frac{d_{S}{ }^{3}}{\left(l_{C}+0.8 d_{C}\right) d_{C}{ }^{2}}\right] I_{C}
$$




$$
\begin{gathered}
\Delta t=\frac{2 \pi \eta\left(I_{C}+0.8 d_{C}\right)^{2} d_{S}\left(1+d_{S} / 2 d_{D}\right)}{V Q} \\
v_{e p} \equiv \frac{l_{C}}{\Delta t}=l_{C}\left[\frac{2 \pi \eta\left(I_{C}+0.8 d_{C}\right)^{2} d_{S}\left(1+d_{S} / 2 d_{D}\right)}{V Q}\right]
\end{gathered}
$$

Here, Ic is the measured ionic current upon the application of a membrane potential (V), and Q is the charge on a particle; $l_{c}, d_{c}, d_{D}$, and ds are the channel length, channel width, Debye length and particle diameter, respectively. For the simplicity, the counter flow effect induced by the counter-ion buildup around the charged nanoparticle was not included. The factor $0.8 \mathrm{~d}_{\mathrm{C}}$ corrects for the so-called "end effect", which becomes significant when the pore diameter is comparable to the pore length [8]. $\mathrm{S}\left(\mathrm{d}_{\mathrm{C}}, \mathrm{ds}_{\mathrm{S}}\right)$ is a correction factor that depends on the relative values of $\mathrm{d}_{\mathrm{C}}$ and $\mathrm{ds}_{\mathrm{s}}$, and is very close to 1 for most cases [11]. From the measured current $\left(\Delta \mathrm{I}_{\mathrm{C}}\right)$ and dwell-time $(\Delta \mathrm{t})$, we determine not only the diameter of a single particle but also the translocation velocity, which is mainly due to electrophoresis in the nanopore.

Figure 1. Schematic of the nanopore device that a single magnetic nanoparticle passes through; (a) a charged magnetic NP in solution moves through the nanopore under both an electric field (electrophoresis) and a magnetic field gradient (magnetophoresis) (b) comparison of the net velocity vector of a charged magnetic NP due to an electric field (left) and to both electric and magnetic fields (right).

(a)
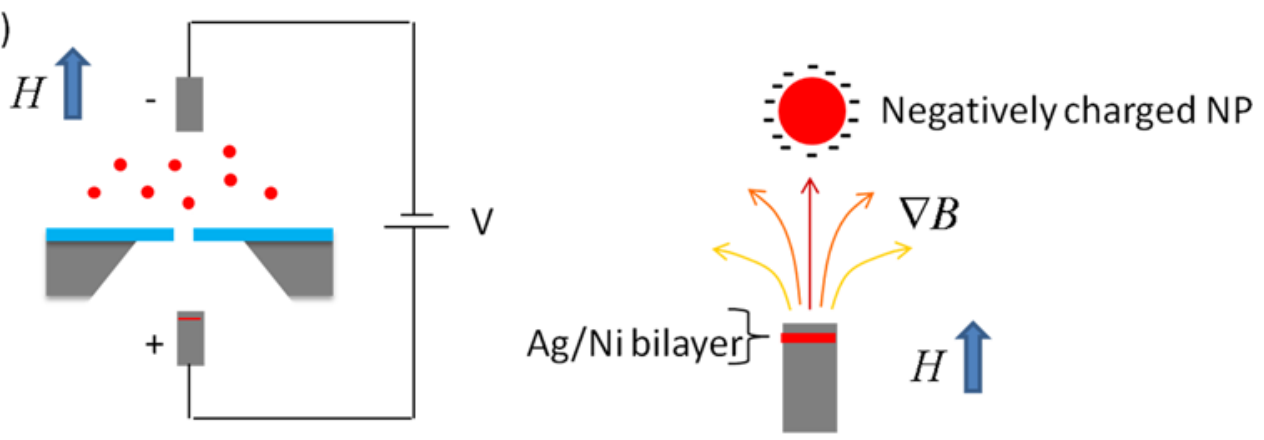

(b)
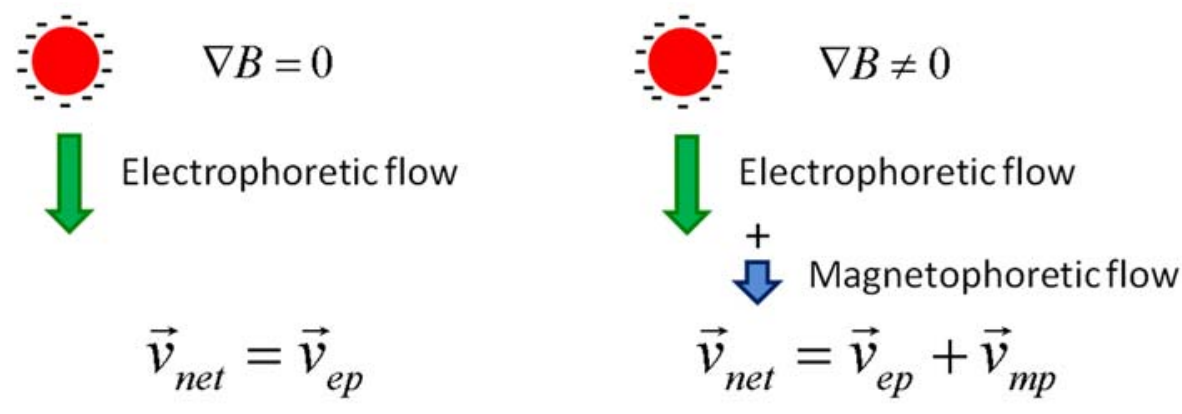

By applying an inhomogeneous magnetic field parallel to the electric field, the MNPs can be pulled in the field direction due to a magnetophoretic flow, which can be simply described by the relation between the magnetic drag force $\left(\mathrm{F}_{\mathrm{mag}}\right)$ and the opposing hydrodynamic drag force $\left(\mathrm{F}_{\mathrm{H}}\right)[12,13]$ :

$$
\vec{F}_{H}=\vec{F}_{\text {mag }}
$$




$$
\begin{gathered}
6 \pi \eta v_{m p} \cdot \frac{d_{S}}{2}=\frac{V \chi}{\mu_{0}}(\vec{B} \cdot \nabla) \vec{B}=\frac{\pi \cdot d_{s}{ }^{3} \cdot \chi}{6 \cdot \mu_{0}}(\vec{B} \cdot \nabla) \vec{B} \\
v_{m p}=\frac{d_{s}{ }^{2} \chi}{18 \eta \mu_{0}}(\vec{B} \cdot \nabla) \vec{B}
\end{gathered}
$$

Here, $\eta$ is the viscosity of the surrounding medium and $v_{\mathrm{mp}}$ is the velocity of a particle due to the magnetophoretic flow. The resultant velocity is linearly proportional to the magnetization of a single MNP. Therefore, magnetization can be obtained by measuring the net velocity ( $\left.v_{\text {net }}\right)$, which is composed of $v_{\mathrm{mp}}$ and $v_{\mathrm{ep}}$.

The basic principle of work is based on the following two factors: (i) electrophoresis: the pull of the MNP into the nanopore with $v_{\text {ep }}$; (ii) magnetophoresis: changing the net velocity $\left(v_{\text {net }}=v_{\text {ep }}+v_{\mathrm{mp}}\right)$. The velocity obtained is converted into the magnetization per particle using Equation (6).

Figure 2. (a), (b) SEM images of a solid state nanopore ( $\mathrm{Si}_{3} \mathrm{~N}_{4}$ membrane on a $\mathrm{Si}$ wafer) which was fabricated by MEMS technology and then drilled by focused ion-beam (FIB) etching; (c) photographic view of the PDMS chamber configuration.

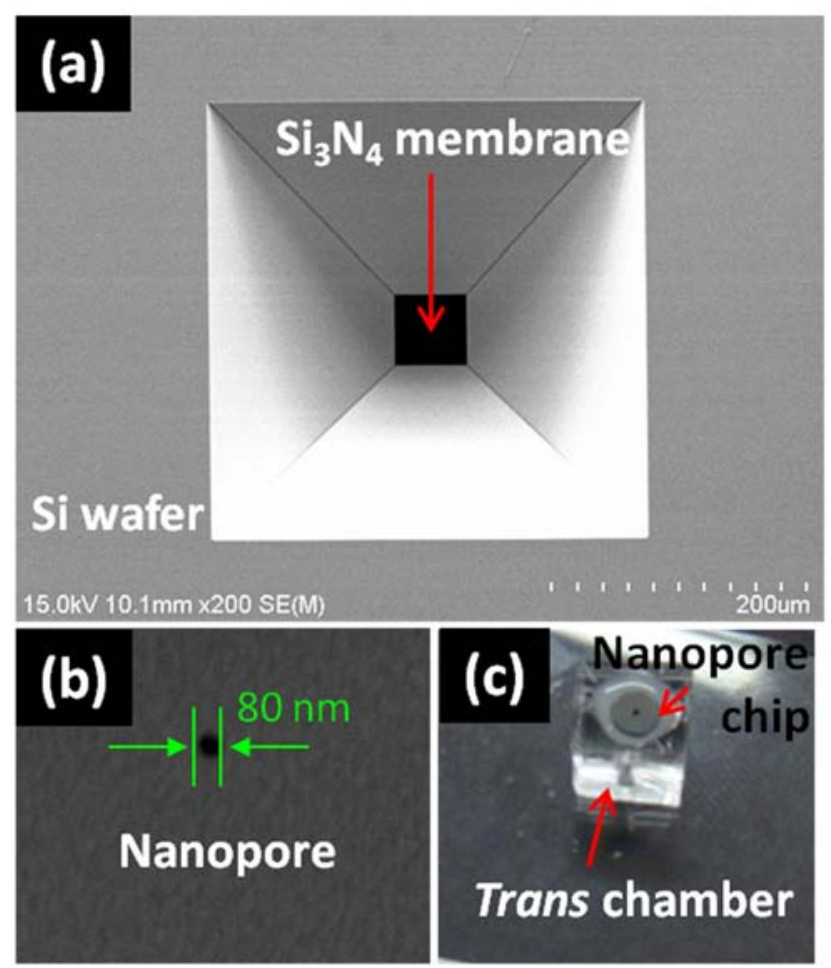

\section{Experiments}

The detector that was used in this study was a solid-state nanopore, which was fabricated in a freestanding silicon nitride $\left(\mathrm{Si}_{3} \mathrm{~N}_{4}\right)$ membrane using conventional MEMS technology (see Figure 2) $[14,15]$. The $\mathrm{lc}_{\mathrm{c}}$, and $\mathrm{dc}_{\mathrm{c}}$ were kept at $30 \mathrm{~nm}$ and $80 \mathrm{~nm}$, respectively. The cis and trans chamber set was fabricated with PDMS (Sylgard 184, Dow Corning, Inc., Midland, MI, USA) and was $1 \mathrm{~mm}$ in diameter. A $0.5 \mathrm{M} \mathrm{KCl}$ solution without buffer was used as a conducting solution and was prepared using deionized water and $\mathrm{KCl}$ powder purchased from Sigma-Aldrich, Co. (St. Louis, MO, USA), followed by filtration through a 0.2 syringe filter (Whatman, Ltd., Piscataway, NJ, USA) prior to use. 
The MNPs that were used in this experiment were obtained from Nanobrick, Inc. (Suwon, Korea) and from Micromod, GmbH (Rostock, Germany) and were negatively charged and tetrabutylammonium hydroxide-stabilized.

The $\mathrm{Si}_{3} \mathrm{~N}_{4}$ nanopore was clamped between the two half chambers, sealed with epoxy, and then placed in the center of a vertical Helmholtz coil in a Faraday cage on a vibration-isolated optical bench. The ionic current was measured in the voltage-clamp mode with an integrated data acquisition system (Axopatch 200B and Digidata 1440A, Molecular Devices, LLC, Sunnyvale, CA, USA). An inhomogeneous magnetic field was generated around the nanopore by magnetic induction from a $\mathrm{Ag} / \mathrm{Ni} / \mathrm{AgCl}$ electrode coupled with the magnetic field in the Helmholtz coil. The magnetic field was measured using a commercial magnetic sensor (Model 410 Gaussmeter, Lake Shore Cryonics, Inc., Westerville, $\mathrm{OH}, \mathrm{USA}$ ) to obtain a spatial distribution of the magnetic field. First, the magnetic sensor was located at the fixed position where the nanopore would be located in the translocation velocity measurements. Then, the $\mathrm{Ag} / \mathrm{Ni} / \mathrm{AgCl}$ electrode was moved precisely from one location to another by a micromanipulator to measure a distance (between the nanopore and the Ni-coated electrode) dependent magnetic fields. Thus, the magnetic field profile near the nanopore was obtained at a given (induced) magnetic field, and then the gradient of the magnetic field at the nanopore was extracted from the measured magnetic field profile. With the information obtained above, we could magnified measure translocation velocity under various magnetic fields, and extract the magnetization-magnetic field (M-B) curve from Equation (6). The $\mathrm{Ag} / \mathrm{Ni} / \mathrm{AgCl}$ electrode was fabricated by e-beam evaporation of $\mathrm{Ni}$ and $\mathrm{Ag}$ on a $\mathrm{AgCl}$ pellet.

Figure 3. A measurement of the ionic current vs. applied voltage through the solid-state nanopore.

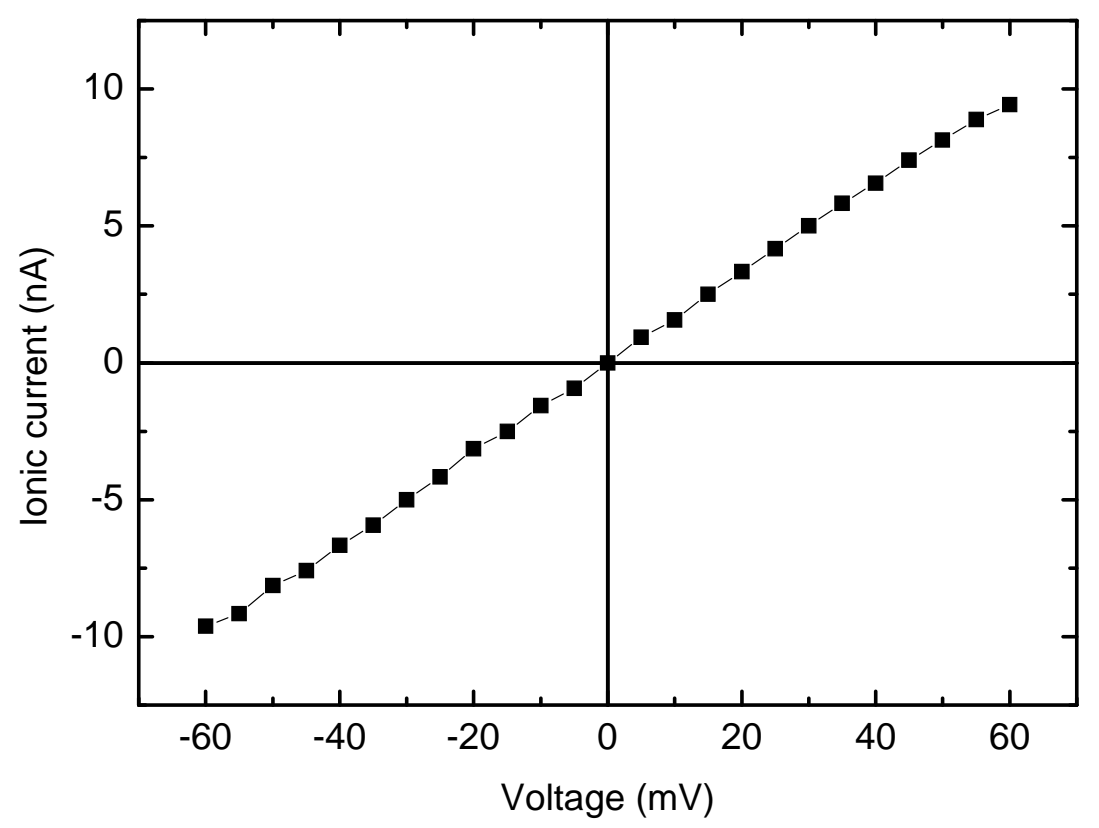


Figure 4. Time-dependent ionic current of charged magnetic NPs in the absence of magnetic field, (a) a global view and (b) a magnified view, and in the presence of a magnetic field, (c) a global view and (d) a magnified view. (e) Distribution of the ionic current blockade amplitude $(\Delta \mathrm{I})$ for $300 \mathrm{sec}$. (f) Distribution of the ionic current blockade dwell time $(\Delta \mathrm{t})$.
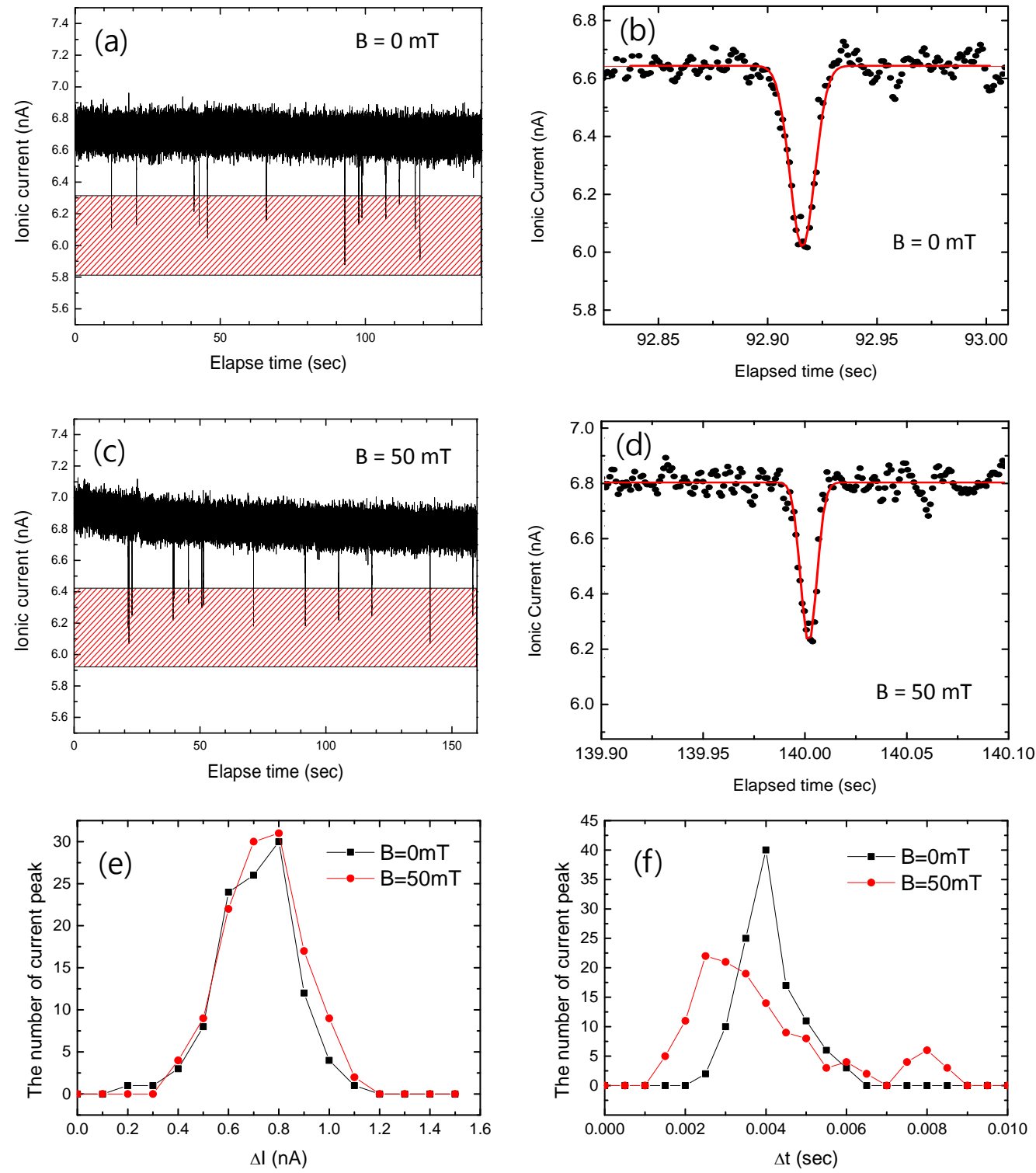

\section{Results}

The Ic-V characteristics of the nanopore device with an $80 \mathrm{~nm}$ pore diameter and a $30 \mathrm{~nm}$ thick silicon nitride membrane are shown in Figure 3. The voltage was swept from $-0.1 \mathrm{~V}$ to $+0.1 \mathrm{~V}$. The experimental and theoretical [16] conductance values were found to be 126 and $285 \mathrm{nS}$, respectively. Thus, the theoretical value $(285 \mathrm{nS})$ is twice as large as the experimental value (126 $\mathrm{nS})$. This inconsistency may be due to the underestimated dimension and the non-ideal shape of the nanopore. The calculation of the conductance used the assumption that the nanopore is cylindrical and has a very smooth wall. However, the actual shape is not cylindrical, but rather is close to hourglass-like [17,18]. Therefore, the effective length is shorter and the radius is smaller than the actual physical diameter 
which could be determined by FE-SEM. Furthermore, the roughness of the nanopore wall effectively reduces the diameter.

Figure 4 shows the time-dependent ionic current (IC vs. $\mathrm{t}$ recording) for $5 \mathrm{~min}$. Hundreds of current blockade events could be observed due to MNP translocation for both $\mathrm{B}=0$ in Figure $4(\mathrm{a}, \mathrm{b})$ and $\mathrm{B} \neq 0$ in Figure 4(c,d). The current blockades that are shown in Figure 4(b,d) have a slightly asymmetric triangular shape, consistent with those of spherical particles in previous works [19]. To obtain $\Delta \mathrm{I}_{\mathrm{C}}$ and $\Delta \mathrm{t}$, the current blockades were simply fitted with a Gaussian curve rather than with multiple peaks because Gaussian fitting is more intuitive for the understanding of the motion of a single sphere. By fitting a hundred current blockade events, histograms of $\Delta \mathrm{I}_{\mathrm{C}}$ and $\Delta \mathrm{t}$ were obtained as shown in Figure $4(\mathrm{e}, \mathrm{f})$, respectively. It should be noted that the average value of $\Delta \mathrm{I}_{\mathrm{C}}$ for $\mathrm{B}=0 \mathrm{mT}$ was the same as that for $\mathrm{B}=50 \mathrm{mT}$, whereas $\Delta \mathrm{t}$ for $\mathrm{B}=50 \mathrm{mT}$ was shortened by approximately $28 \%$. The histograms reflect the fact that $\Delta \mathrm{I}_{\mathrm{C}}$ is only dominantly governed by the size of a single particle from Equation (1), while $\Delta \mathrm{t}$ stems from the velocity that is driven not only by the electrophoresis from Equation (3) but also by the magnetophoresis from Equation (6). Therefore, the size and magnetization of a single MNP could be determined from the $\Delta \mathrm{I}_{\mathrm{C}}$ and $\Delta \mathrm{t}$ histograms, respectively.

We compared the size distribution from $\Delta \mathrm{I}_{\mathrm{C}}$ with the one taken from dynamic light scattering (DLS) as shown in Figure 5. The distribution from the DLS experiment, which is shown in Figure 5(a), revealed that the most frequent size and the average value are $33 \mathrm{~nm}$ and $31.3 \mathrm{~nm}$, respectively, which are very close to the anticipated numbers. The distribution from $\Delta \mathrm{I}_{\mathrm{C}}$, which is shown in Figure 5(b), was in a good agreement with that from DLS. The error likely arises from the background current noise.

Figure 5. Size distribution of the magnetic nanoparticle obtained from (a) the dynamic light scattering (DLS) method and (b) the ionic current blockade.

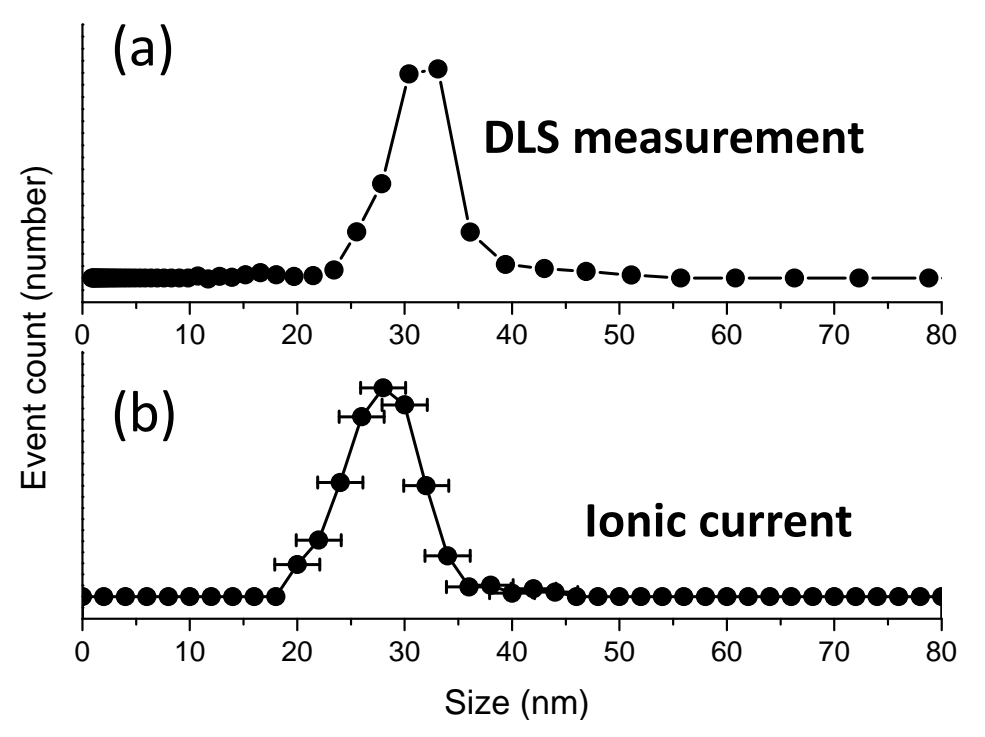

To explicitly verify the effect of the magnetic force on the velocity, the translocation velocity was measured as a function of the external magnetic field, as shown in Figure 6(a). It can be seen that the velocity increased with increasing external magnetic field. This tendency indicates that the change in the velocity is mainly driven by the magnetic force between the external field and the MNPs. It should be noted that the histograms broadened with an increase in the magnetic field. The dependence of this broadening on the external magnetic field could be explained by the existence of slightly different 
magnetizations among the MNPs, which would lead to a variation in the slope of the M-B curve (which is proportional to the magnetic susceptibility). This variation in the susceptibility would enable the distribution of the velocity to broaden with an increase in the external field. Figure 6(b) shows a comparison of the M-B curves from the velocity measurements with that from VSM measurements. The velocity was converted to the magnetization per particle using Equation (6) in the Section 2. For high magnetic field regime at $\mathrm{B}=100 \mathrm{mT}$, the gradient of the magnetic field on the order of $100 \mathrm{~T} / \mathrm{m}$ was obtained from the field vs. distance measurement as described in the Section 3. The susceptibility from the ionic current was linearly proportional to the magnetic field in the range of $\mathrm{B}<100 \mathrm{mT}$, and was approximately half the value of the VSM result of 1.6. The discrepancy in the absolute value of the susceptibility might be due to errors in the effective parameter estimations including the "end effect", but is still under investigation.

Figure 6. (a) Velocity distribution of the magnetic nanoparticles under various magnetic fields. (b) M-B curves obtained from VSM measurements (open circle) and ionic current measurements (closed circle).
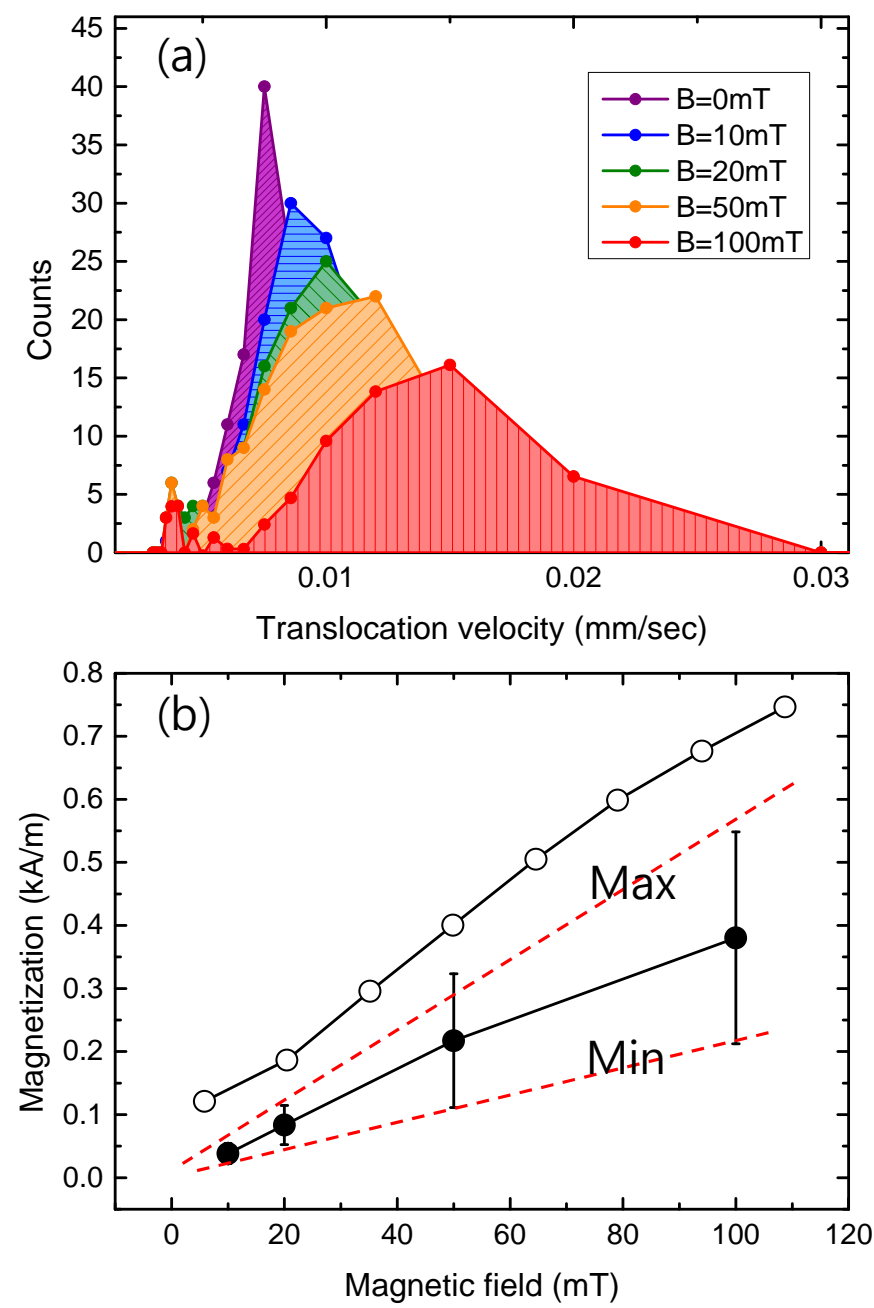

\section{Conclusions}

We have investigated the properties of magnetic nanoparticles translocating through a solid-state nanopore in the presence of an inhomogeneous magnetic field. By measuring and analyzing the ionic 
current blockade, the translocation velocity of a nanoparticle passing through a nanometer-scale channel was found to be linearly proportional to the magnetization of the single magnetic nanoparticle. We have also shown the possibility of using this method to analyze the magnetic properties of hundreds of individual nanoparticles within a few minutes. We expect that this method will find a number of interesting applications, not only for characterizing the magnetic properties of inorganic nanoparticles but also for manipulating and analyzing organic and biological molecules that have been hybridized with magnetic nanoparticles.

\section{Acknowledgments}

This work was supported by the Industrial Strategic Technology Development Program, (10037410-2011-02, Development of Next Generation DNA Sequencer), funded by the Ministry of Knowledge Economy (MKE, Korea). This research was also supported by the grant (2012-P3-14) from the Advanced Institutes of Convergence Technology (AICT) and the Ministry of Education, Science and Technology of Korea as Global Frontier Project (CISS-2012366054204).

\section{Conflict of Interest}

The authors declare no conflict of interest.

\section{References}

1. Koh, I.; Josephson, L. Magnetic nanoparticle sensors. Sensors 2009, 9, 8130-8145.

2. Haun, J.B.; Yoon, T.-J.; Lee, H.; Weissleder, R. Magnetic nanoparticle biosensors. Wiley Interdiscip. Rev. Nanomed. Nanobiotechnol. 2010, 2, 291-304.

3. Biswal, S.L.; Gast, A.P. Micromixing with linked chains of paramagnetic particles. Anal. Chem. 2004, 76, 6448-6455.

4. Sandhu, A.; Kumagai, Y.; Lapicki, A.; Sakamoto, S.; Abe, M.; Handa, H. High efficiency Hall effect micro-biosensor platform for detection of magnetically labeled biomolecules. Biosens. Bioelectron. 2007, 22, 2115-2120.

5. Park, S.Y.; Handa, H.; Sandhu, A. Magneto-optical biosensing platform based on light scattering from self-assembled chains of functionalized rotating magnetic beads. Nano Lett. 2010, 10, 446-451.

6. Baselt, D.R.; Lee, G.U.; Natesan, M.; Metzger, S.W.; Sheehan, P.E.; Colton, R.J. A biosensor based on magnetoresistance technology. Biosens. Bioelectron. 1998, 13, 731-739.

7. Park, S.Y.; Handa, H.; Sandhu, A. Determination of inter-molecular forces by magneto-optical transmittance of molecule-covered superparamagnetic particles in solution. IEEE Trans. Magn. 2010, 46, 1409-1411.

8. Wang, J.P. FePt Magnetic nanoparticles and their assembly for future magnetic media. Proc. IEEE 2008, 96, 1847-1863.

9. Choi, J.W.; Oh, K.W.; Thomas, J.H.; Heineman, W.R.; Halsall, H.B.; Nevin, J.H.; Helmicki, A.J.; Henderson, H.T.; et al. An integrated microfluidic biochemical detection system for protein analysis with magnetic bead-based sampling capabilities. Lab Chip 2002, 2, 27-30. 
10. Overbeek, J.T.G.; Wiersema, P.H. Interpretation of Electrophoretic Mobilities. In Electrophoresis: Theory, Methods and Applications; Bier, M., Ed.; Academic Press: New York, NY, USA, 1967; Volume 2, pp. 1-52.

11. Deblois, R.W.; Bean, C.P. Counting and Sizing of Submicron Particles by Resistive Pulse Techniques. Rev. Sci. Instrum. 1970, 41, 909-916.

12. Pamme, N. Continuous flow separations in microfluidic devices. Lab Chip 2007, 7, 1644-1659.

13. Häfeli, U.O.; Lobedann, M.A.; Streingroewer, J.; Moore, L.R.; Riffle, J. Optical method for measurement of magnetophoretic mobility of individual magnetic microspheres in defined magnetic field. J. Magn. Magn. Mater. 2005, 293, 224-239.

14. Storm, A.J.; Chen, J.H.; Ling, X.S.; Zandbergen, H.W.; Dekker, C. Fabrication of solid-state nanopores with single-nanometre precision. Nat. Mater. 2003, 2, 537-540.

15. Krapf, D.; Wu, M.Y.; Smeets, R.M.M.; Zandbergen, H.W.; Dekker, C.; Lemay, S.G. Fabrication and characterization of nanopore-based electrodes with radii down to $2 \mathrm{~nm}$. Nano Lett. 2006, 6, 105-109.

16. Kowalczyk, S.W.; Grosberg, A.Y.; Rabin, Y.; Dekker, C. Modeling the conductance and DNA blockade of solid-state nanopores. Nanotechnology 2011, 22, 315101.

17. Wu, M.Y.; Smeets, R.M.M.; Zandbergen, M.; Ziese, U.; Krapf, D.; Batson, P.E.; Dekker, N.H.; Dekker, C.; Zandbergen, H.W. Control of shape and material composition of solid-state nanopores. Nano Lett. 2009, 9, 479-484.

18. Haynes, W.M., Ed. CRC Handbook of Chemistry and Physics, 91st ed.; CRC Press: Boca Raton, FL, USA, 2010.

19. Ito, T.; Sun, L.; Crooks, R.M. Simultaneous determination of the size and surface charge of individual nanoparticles using a carbon nanotube-based coulter counter. Anal. Chem. 2003, 75, 2399-2406.

(C) 2013 by the authors; licensee MDPI, Basel, Switzerland. This article is an open access article distributed under the terms and conditions of the Creative Commons Attribution license (http://creativecommons.org/licenses/by/3.0/). 